\title{
A novel EMG interface for individuals with tetraplegia to pilot robot hand grasping
}

\author{
W. Tigra ${ }^{1,2 *}$, B. Navarro ${ }^{3,4}$, A. Cherubini ${ }^{3}$, X. Gorron ${ }^{3}$, A. Gelis ${ }^{5}$, C. Fattal ${ }^{6}$, D. Guiraud ${ }^{1}$, C. Azevedo Coste $^{1}$
}

\begin{abstract}
This article introduces a new human-machine interface for individuals with tetraplegia. We investigated the feasibility of piloting an assistive device by processing supra-lesional muscle responses online. The ability to voluntarily contract a set of selected muscles was assessed in five spinal cord-injured subjects through electromyographic (EMG) analysis. Two subjects were also asked to use the EMG interface to control palmar and lateral grasping of a robot hand. The use of different muscles and control modalities was also assessed. These preliminary results open the way to new interface solutions for high-level spinal cord-injured patients.
\end{abstract}

Index terms - Control, electromyographic (EMG), grip function, robot hand, tetraplegia.

\section{INTRODUCTION}

Consequences of complete spinal cord injury (SCI) are often devastating for patients. This observation is particularly true for trauma at cervical levels (tetraplegia), since this impedes the use of the four limbs. Indeed, a complete SCI prevents any communication between the central nervous system and the sub-lesional peripheral nervous system, which receives no cervical commands. However, moving paralyzed limbs after such trauma is still possible, as for example when sufficient electric current is applied. Cells (neurons or myocytes), are then excited and generate the action potentials responsible for muscle contraction [1], [2], [3], [4]. Nevertheless, the interaction of the tetraplegic person with his/her electrical stimulation device, to control the artificial contractions and achieve a given task at the desired instant, is still problematic. The reason is that both the range of possible voluntary movements, and the media available to detect intention, are limited. Various interface types have therefore been tested in recent years. For lower limbs, these interfaces include push buttons on walker handles in assisted-gait [5], accelerometers for movement detection in assisted-sit-to-stand [6], electromyography (EMG) [7] and evoked-electromyography (eEMG) [8] and, most recently, brain computer interfaces (BCI) [9]. For upper limbs (restoring hand movement), researchers have proposed the use of breath control, joysticks, electromyography (EMG) [10], shoulder movements [11], and voluntary wrist extension [12]. In this last work, a wrist osseointegrated Hall effect sensor implant provided the functional electrical stimulation (FES) of a hand neuroprosthesis. Keller et al. proposed using surface EMG from the deltoid muscle of the contralateral

\footnotetext{
* Corresponding author wafa.tigra@inria.fr

${ }^{1}$ INRIA, LIRMM, Montpellier, France.

${ }^{2}$ MXM, Sophia-Antipolis, France

${ }^{3}$ IDH LIRMM, Université Montpellier - CNRS, Montpellier, France.

${ }^{4}$ PRISME Laboratory, University of Orléans - INSA CVL, Bourges, France.

${ }^{5}$ Propara Center, Montpellier, France.

${ }^{6}$ CRF COS DIVIO, Dijon, France.
}

arm to stimulate the hand muscles [13]. In [14], the EMG signal from the ipsilateral wrist extensor muscles was used to pilot a hand neuroprosthesis. An implanted device [15] took advantage of the shoulder and neck muscles to control the FES applied to the arm and hand muscles. EMG signals were also used to control an upper limb exoskeleton in [16].

Orthotics and FES can be effective in restoring hand movements, but the piloting modalities are often unrelated to the patient's level of injury and remaining motor functions, making the use of these devices somewhat limited. We believe that poor ergonomics and comfort issues related to the piloting modes also explain this low usage. In this paper, we therefore present a control modality closely linked to the patients remaining capacities in the context of tetraplegia.

We propose here to evaluate the capacity and comfort of contracting supra-lesional muscles [17], and assess the feasibility of using EMG signals as an intuitive mode of controlling of functional assistive devices for upper limbs. In this preliminary study, we focus on the comfort and capacity for contracting four upper limb muscles (trapezius, deltoid, platysma and biceps) in individuals with tetraplegia. We then investigate the feasibility of using these contractions to control the motions of a robot hand.

A robot hand was preferred to conventional grippers since it allows manipulators or humanoids to handle complex shaped parts or objects that were originally designed for humans, at the cost of more sophisticated mechanical designs and control strategies [18],[19]. Recently, robot hand usage has been extended to the design of prostheses for amputees, under the control of brain-computer interfaces [20], or EMG signals [21],[22],[23],[24]. However, to our knowledge, surface EMG signals (in contrast to neural signals [25],[26]) have never been used by tetraplegic individuals to pilot robot hands. CWRU [7], for example, used EMG signals to pilot the patient's own hand through FES, whereas Dalley et al. [27] used EMG within a finite state machine to control a robot hand, but with healthy subjects. Furthermore, in most of the cited works, a single motor was used to open or close a finger, a design constraint that impedes precise hand postures and grasps. Using a fully dexterous robot hand allowed us to further investigate the possibilities of an EMG interface to control different grasping modalities owing to the visual feedback provided by the robot hand. Furthermore, the dimensions and degrees of freedom are very close to those of the human hand, therefore providing the user an intuitive representation of the final movement that he/she can control with, for example, FESbased hand movement restoration. The goal of the study was two-fold: (i) to assess the ability of tetraplegic patients to pilot a robot hand device via muscle contractions even though the contractions are not functional. The EMG signals came from 
supra-lesional muscles that can be very weak and unable to produce any movement; and (ii) to compare different control modalities.

In the following section, we present the protocol and experimental setup. We then present the results on the efficacy and comfort of the continuous or graded contraction of different muscles, along with details on the participants capacity to pilot the robot hand using these contractions.

\section{MAterial AND Methods}

\section{A. Subjects and selected muscles}

The study was conducted during scheduled clinical assessments at the Propara Neurological Rehabilitation Center in Montpellier, France. Thus, the experiments had to be of limited duration. The subjects were informed verbally and in writing about the procedure and gave their signed informed consent according to the internal rules of the medical board of the Centre Mutualiste Neurologique Propara. The experiments were performed with five tetraplegic male subjects with lesional levels between C5 and C7 (see Table I). Subject 2 had undergone muscle-tendon transfer surgery at the time of inclusion.

Surface BIOTRACE Electrodes (Controle graphique S.A, France) were used for EMG recordings. Pairs of surfacerecording electrodes $(1 \mathrm{~cm}$ distance) were positioned above the four muscles on each body side. Subjects did not receive any pre-training before these experiments. They were only instructed on the movements for contracting the various muscles.

TABLE I

SUBJECT CHARACTERISTICS

\begin{tabular}{|c|c|c|c|c|}
\hline Subject ID & Age & $\begin{array}{c}\text { Level of the } \\
\text { lesion }\end{array}$ & $\begin{array}{c}\text { AIS }^{1} \\
\text { score }\end{array}$ & $\begin{array}{c}\text { Interval time } \\
\text { since SCI (years) }\end{array}$ \\
\hline 1 & 33 & C6 & A & 4 \\
\hline 2 & 35 & C7 & A & 13 \\
\hline 3 & 21 & C7 & A & 0,5 \\
\hline 4 & 48 & C5 & A & 32 \\
\hline 5 & 45 & C6 & C & 4 \\
\hline
\end{tabular}

As the muscles selected to control hand grasp devices are likely to be used in a daily context by tetraplegic subjects, these muscles should be under voluntary control. The targeted tetraplegic patients had no muscle under voluntary control under the elbow. The use of facial muscles to pilot a hand grasp device has never been studied because social acceptability would probably be problematic. In addition, muscle synergies were sought (e.g., hand closing could be linked to elbow flexion, as performed via the biceps or deltoid muscle). For these reasons, we chose to study the EMG activity of four upper arm muscles (right and left): the middle deltoid, the superior trapezius, the biceps and the platysma. Nevertheless,

\footnotetext{
${ }^{1}$ The ASIA (American Spinal Injury Association) Impairment Scale (AIS) classifies the severity (i.e. completeness) of a spinal cord injury. The AIS is a multi-dimensional approach to categorize motor and sensory impairment in individuals with SCI. It identifies sensory and motor levels indicative of the most rostral spinal levels, from A (complete SCI) to E (normal sensory and motor function) [28].
}

there were slight differences in these eight muscles based on each subjects remaining ability. EMG signals are initially recorded on the ipsilateral and contralateral sides of the dominant upper limb. Yet, patients 1 and 3 showed signs of fatigue and they did not use the contralateral (left) limb. The superior trapezius, middle deltoid, biceps and platysma muscles of the ipsilateral side of the dominant (right) upper limb were thus studied for these subjects. For subjects 2 and 4, both (left and right) superior trapezii, middle deltoids, bicepses, and platysmas were considered. For patient 5, the deltoid was remplaced by the middle trapezius, which has a similar motor schema, since strong electrocardiogram signals were observed on the deltoid EMG signal. To guarantee that the selected EMG would not impede available functionality, the patients' forearms were placed in an arm brace and EMGs signals were recorded with quasi-isometric movements.

\section{B. EMG processing}

Surface EMG signals were recorded with an insulated National Instrument acquisition card NI USB 6218, 32 inputs, 16-bit (National Instruments Corp., Austin, TX, USA). BIOVISION EMG amplifiers (Wherheim, Germany) were used, with gain set to 1000 . The acquisition card was connected to a batteryrun laptop computer. The acquisition was made at $2.5 \mathrm{kHz}$.

For the first three subjects, the data processing was offline: EMG data were filtered with a high-pass filter $(20 \mathrm{~Hz}$, fourthorder Butterworth filter, 0 phase). Then, a low-pass filter was applied to the absolute value of the EMG to obtain its envelope ( $2 \mathrm{~Hz}$, fourth-order Butterworth filter). The data processing was online for the other two subjects in order to control the robot hand motion. We applied the same filtering except for the first filter, which had a non-zero phase. In all cases, the filtered EMG signal is denoted with $s(t)$.

A calibration phase was performed for each muscle's EMG. Subjects were asked to first relax the muscle and then to strongly contract it. The corresponding EMG signals were stored and post-processed to obtain the maximum envelope. The thresholds were then set as a proportion of the normalized value of the EMG signal (value for a maximal contraction =1). The high and low thresholds were experimentally determined to $s_{L}=0.3 \pm 0.1$ and $s_{H}=0.44 \pm 0.14$ through the calibration process, in order to avoid false detection against noise, while maintaining them as low as possible, to require only a small effort from the patient. These thresholds, $s_{L}$ and $s_{H}\left(s_{H}>s_{L}>0\right)$, were used to trigger the states of the robot hand finite state machine (FSM), as explained below.

FSMs have been used in some myoelectric control studies, mostly on healthy or amputees subjects, but never with tetraplegic subjects [27]. In our study, the goal was to determine whether the muscles in the immediate supra-lesional region could be used by tetraplegic patients to control a robot hand. We relied on myoelectric signals, even from very weak muscles that were unable to generate torque sufficient to pilot the hand. As we controlled only three hand states through event-triggered commands, an FSM was appropriate. On the contrary, EMG pattern recognition is mostly used to progressively pilot several hand movements from many sensors. 
TABLE II

DESCRIPTION OF THE FIVE HAND CONTROL MODES

\begin{tabular}{|l|l|}
\hline Mode $\mathrm{n}^{\mathrm{o}}$ & Description \\
\hline 1 & $\begin{array}{l}\text { Continuous muscle contraction provokes } \\
\text { grasping. When the muscle is relaxed, the } \\
\text { hand opens. }\end{array}$ \\
\hline 2 & $\begin{array}{l}\text { A first contraction of } 2 \mathrm{~s} \text { triggers grasping. } \\
\text { The hand remains closed even when the } \\
\text { muscle is relaxed. The next } 2 \mathrm{~s} \text { contraction } \\
\text { triggers hand opening. }\end{array}$ \\
\hline 3 & $\begin{array}{l}\text { Grasping is related to EMG amplitude } \\
\text { (stronger EMG signal leads to tighter } \\
\text { closure). When the muscle is relaxed, the } \\
\text { hand opens. }\end{array}$ \\
\hline 4 & $\begin{array}{l}\text { Contracting (for } 2 \mathrm{~s} \text { ) first muscle 1 causes } \\
\text { palmar pinch (palmar grasping); then, the } \\
\text { hand can be opened by contracting (for 2 s) } \\
\text { muscle 2. Instead, contracting first (for 2 s) } \\
\text { muscle } 2 \text { causes key-grip (lateral grasping), } \\
\text { followed by hand opening if muscle 1 is } \\
\text { contracted (for } 2 \text { s). }\end{array}$ \\
\hline 5 & $\begin{array}{l}\text { Contraction of muscle 1 causes a palmar } \\
\text { pinch, whereas contraction of muscle } 2 \\
\text { causes key-grip. In both cases, to stop the } \\
\text { closure, subjects must stop muscle contraction } \\
\text { (cf. Fig. 1). }\end{array}$ \\
\hline
\end{tabular}

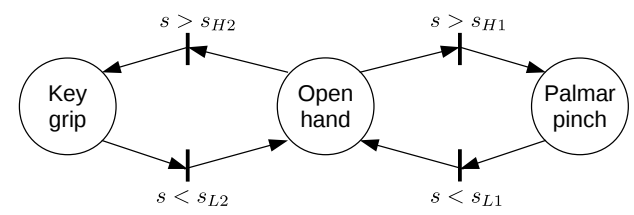

Fig. 1. Finite state machine used to control the hand in Mode 5.

\section{Robot hand control}

We chose to use the robot hand since it gives patients much more realistic feedback on task achievement (via grasp of real objects) compared to a virtual equivalent (e.g., a simulator). With a real (yet robot) hand, patients can perform the task as if FES had been used on their hand. The Shadow Dexterous Hand (Shadow Robot Company, London, UK) closely reproduces the kinematics and dexterity of the human hand. The model used here is a right hand with 19 cable-driven joints (denoted by angle $\mathbf{q}_{i}$ for each finger $i=1, \ldots, 5$ ): two in the wrist, four in the thumb and little finger, and three in the index, middle and ring fingers. Each fingertip is equipped with a BioTac tactile sensor (SynTouch, Los Angeles, CA, USA). These sensors mimic human fingertips by measuring pressure, vibrations and temperature. The hand is controlled through ROS ${ }^{1}$, with the control loop running at $200 \mathrm{~Hz}$.

In this work, the hand could be controlled in five alternative modes, shown in Table III. Each mode corresponds to a different FSM, and the transitions between states are triggered by muscle contractions and relaxations. Three hand states were used: open hand, palmar pinch, and key-grip (see Fig 2). Unlike the other modes, mode 3. is not an "all-or-nothing" closing, but allows progressive closing, according to the amplitude of the EMG signal. To begin grasping, contraction has to

\footnotetext{
${ }^{1}$ http://www.ros.org
}

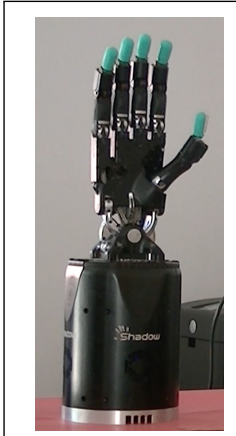

(a)

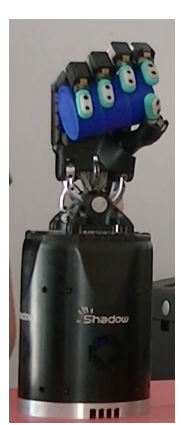

(b)

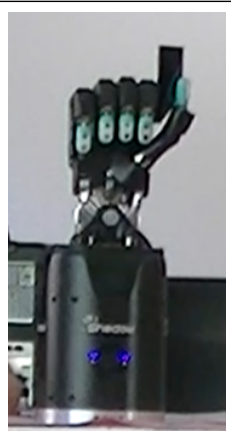

(c)
Fig. 2. Different states of the robot hand: (a) open hand, (b) palmar pinch (palmar grasping), (c) Key-grip (lateral grasping).

be above the first chosen threshold, and then the finger position is proportional to the EMG envelope amplitude. One muscle is monitored in modes 1 to 3 , and two muscles in modes 4 and 5 (see Table II). Hysteresis was used: we considered a muscle contracted if $s(t)>s_{H}$ and relaxed if $s(t)<s_{L}$. For $s(t) \in\left[s_{L}, s_{H}\right]$, the muscle (hence, hand) state is not changed. In modes 1-3, only one - predetermined - grasp (palmar) was used, whereas in modes 4 and 5 , the user was able to change the grasp (palmar/lateral) type online via the EMG signal. Each state was characterized by the five finger target joint values, $\mathbf{q}_{i}^{*}$. In all modes, except for mode 3 , these were pretuned offline to constant values (corresponding to open and closed configurations).In mode 3 , however, the desired finger position $\mathbf{q}_{i}^{*}$ was obtained by interpolating between open and closed positions $\left(\mathbf{q}_{i}^{o}\right.$ and $\left.\mathbf{q}_{i}^{c}\right)$ :

$$
\mathbf{q}_{i}^{*}=\mathbf{q}_{i}^{o}\left(1+e\left(\mathbf{q}_{i}^{c}-\mathbf{q}_{i}^{o}\right)\right),
$$

with $e$ the contraction level, normalized between 0 (no contraction) and 1 (full contraction):

$$
e= \begin{cases}1 & \text { if } s>s_{H} \\ 0 & \text { if } s<s_{L} \\ \frac{s-s_{L}}{s_{H}-s_{L}} & \text { otherwise }\end{cases}
$$

We now outline how the target values $\mathbf{q}_{i}^{*}$ were attained.

For the two grasping states, finger motion should stop as soon as contact with the grasped object occurs. To detect contact on each fingertip $i$, we use the pressure measurement $P_{i}$ on the corresponding BioTac. At time $t$, the contact state (defined by the binary value $C_{i}(t)$ ) is detected by a hysteresis comparator over $P_{i}$ :

$$
C_{i}(t)= \begin{cases}1 & \text { if } P_{i}>P_{H}, \\ 0 & \text { if } P_{i}<P_{L} \text { or } t=0, \\ C_{i}(t-T) & \text { otherwise. }\end{cases}
$$

Here, $P_{H}$ and $P_{L}\left(P_{H}>P_{L}>0\right)$ are the pre-tuned high and low thresholds at which $C_{i}$ changes, and $T$ is the sampling period. For the open hand state, we do not account for fingertip contact, and keep $C_{i}(t)=0$.

For all three states, an online trajectory generator (OTG) is used to generate the joint commands $\mathbf{q}_{i}$, ensuring smooth 
motion of each finger to its target value $\mathbf{q}_{i}^{*}$. The commands depend on the contact state:

$$
\mathbf{q}_{i}(t)= \begin{cases}O T G\left(\mathbf{q}_{i}(t-T), \mathbf{q}_{i}^{*}, \dot{\mathbf{q}}_{i}^{M}\right), & \text { if } C_{i}(t)=0 \\ \mathbf{q}_{i}(t-T) & \text { otherwise }\end{cases}
$$

with $\dot{\mathbf{q}}_{i}^{M}$ the vector of - known - maximum motor velocities allowed for the joints of finger $i$. Each finger is controlled by a separate OTG, in order to stop only the ones in contact. As OTG, we used the Reflexxes Motion Library ${ }^{2}$

\section{Experimental protocols}

The experiments were performed through two successive protocols at two different times and with two different sets of patients to limit the duration of the session within their clinical assessment. The first time (protocol A, subjects 1,2 and 3, Fig. 3 , we checked whether the patients could contract each muscle (assumed to be supra-lesional but not far from the lesion) with a sufficient level of EMG. The second time (protocol B, subjects 4 and 5, Fig. 3 we tested their ability to control the robot hand without previous practice so visual feedback (from observing the hand) was added to the proprioceptive feedback (subjects 4 and 5). Both protocols are described below.

1) Protocol A - EMG alone:

This protocol evaluated the subjects' capacity to voluntarily control the different muscles and the comfort and ease of contraction (Fig. 3). Each task was performed only once since the objective was achieved at the first attempt, thereby confirming the easiness of command. Moreover, warm-up was not necessary, since the muscles were not used to output torque but only to generate usable EMG. For each muscle, the subjects performed two tasks:

1) maintain maximum contraction for 15 seconds,

2) successively maintain three levels of contraction (low, medium, high), each for 5 seconds.

\section{2) Protocol B - EMG driving robot hand motion:}

For this second protocol, muscle contractions controlled the robot hand motion (see Fig. 4). Protocol B was thus composed of two consecutive parts: individual, and preferred muscle assessment.

\section{a) Individual muscle assessment:}

In the first part of protocol $\mathrm{B}$, individual muscle contractions were assessed through three tasks.

T1) calibrate: $s_{L}$ and $s_{H}$ are set,

T2) maintain maximum contraction for 5 seconds,

T3) maintain contraction as long as possible (after the minimum of 15 seconds.

In tasks 2 and 3, the contraction level had the empirically defined threshold $s_{H}$. After each muscle assessment, the subject was asked to assess the comfort, fatigue and ease of contraction efforts through a questionnaire. The questionnaire was inspired by the ISO 9241-9 standard on "Ergonomics of non-keyboard input devices." Once all eight muscles were

\footnotetext{
${ }^{2}$ http://www.reflexxes.ws
}
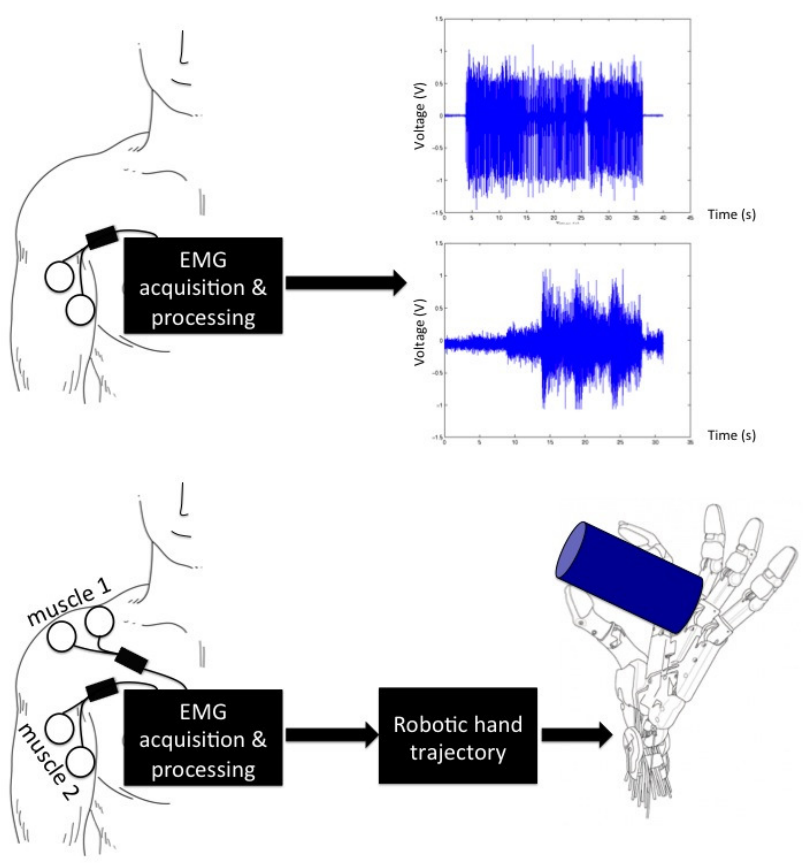

Fig. 3. Top: Principle of EMG recording and analysis (protocol A). Bottom: Principle of robot hand control through EMG signals. (protocol B).

tested, the subjects were asked to select the two preferred muscles. These two muscles were then taken into account to evaluate the different robot hand control modes in the second part of the protocol.

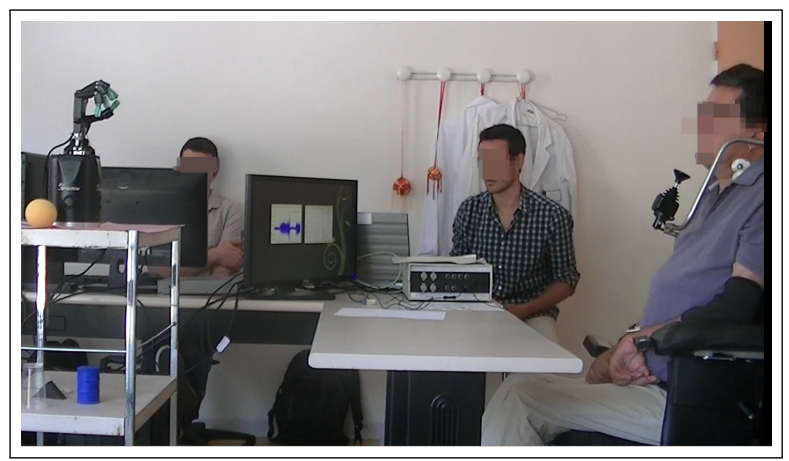

Fig. 4. Protocol B: setup description and upper arm positioning during EMG recordings.

\section{b) Preferred muscle assessment:}

Two muscles were selected among the eight, based on subjective patients assessments. The choice of preferential muscles was up to the patient, with the constraint that these two muscles must be on the same side. All five modes of robot hand control (shown in Table III) were tested and evaluated.

For mode 5, the subject was instructed to select contraction muscle 1 or 2 (i.e., either palmar or lateral grasping), depending on the object randomly presented by the experimenter. Two objects were presented to the subject, one with a cylindrical or spherical shape requiring palmar 
grasping, the other with a triangular prism shape requiring lateral grasping. The subject had to trigger the correct closure of the robot hand through the contraction of the appropriate muscle to grasp the presented object. Each type of prehension was tested at least five times during the 11 randomized trials.

\section{RESULTS}

\section{A. EMG Results}

We analyzed EMG data from continuous (Fig. 5 (a) and Fig. 5 (b)) and graded (Fig. 5 (c) and Fig. 5 (d)) muscle contractions. Data on each subject's ability to contract the

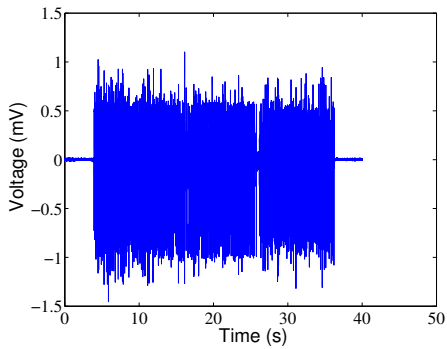

(a)

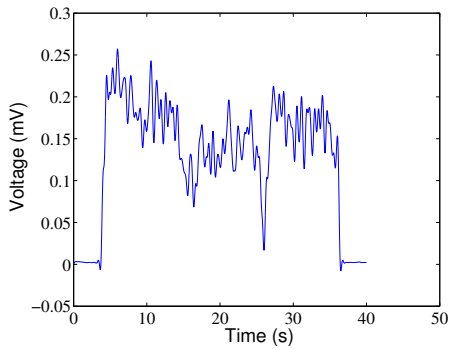

(b)
3 successive biceps muscle contractions (Subj. 4).

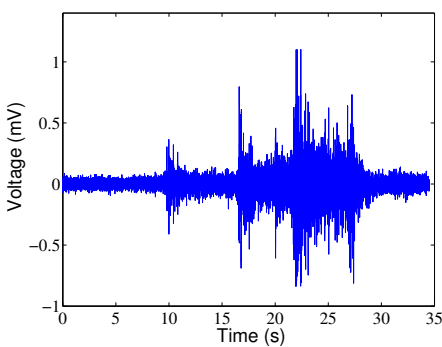

(c)

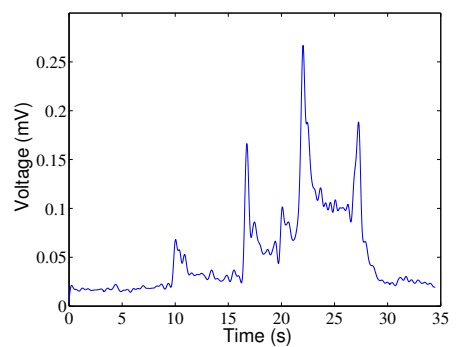

(d)
Graded contraction of sup. trapezius muscle (Subj. 3).

Fig. 5. Example of muscle contractions observed in SCI subjects. Raw signal (a and c), filtered signal ( $b$ and $d)$.

different muscles is presented in Table III. All subjects were able to individually contract the eight muscles on demand for at least 7 seconds, except subject 1 for biceps (no voluntary contraction was visible in the EMG signal).

Interestingly, a contraction could be extracted from the EMG signals even for very weak muscles. This is illustrated in Fig. 5 (a) and Fig. 5 (b), where a voluntary sustained contraction of the subject's left biceps can be seen. He was able to maintain his contraction for more than 30 seconds. Although this subject presented a C5 lesion with non-functional biceps activity (no elbow flexion), this very weak EMG activity of the biceps could still be turned into a functional command to pilot a device.

Among our five patients, there was only one case where a very weak muscle produced a functional EMG signal. This muscle had an MRC $^{1}$ score of 1 . For all other muscles with EMG activity, the MRC score was $\geq 3$. For protocol A

\footnotetext{
${ }^{1}$ The MRC (Medical Research Council) Scale assesses muscle power in patients with peripheral nerve lesions from 0 (no contraction) to 5 (normal power).
}

(subjects 1-3), we present in Table [V] the ability to grade muscle contraction. The three subjects were able to achieve the three levels of contraction (low, medium and high). The biceps of subject 1 was not tested here, as continuous voluntary contraction was not visible in the EMG signal. In Fig. 5 (c) and Fig. 5(d), we present an example of a trial from subject 3. He was able to perform an isometric graded contraction of his superior trapezius muscle, but had difficulties holding the contraction for more than 5 seconds. The amplitude of contraction was increased by a factor of seven from $17.3 \pm 1.9$ (rest level), $34.3 \pm 14.9$ (low contraction), $43.7 \pm 36.1$ (middle contraction) to $78.7 \pm 38.5$ (high contraction). In protocol $\mathrm{B}$, the subjects were able to maintain the contraction of each of the tested muscles.

\section{B. Hand results}

The tasks (e.g., holding the object in the robot hand for $5 \mathrm{~s}$ ) were successfully achieved with each of the tested muscles. Among the tested modes, mode 2 was the favorite mode for subject 4. Mode 1 was the favorite mode for subject 5. Regarding the preferential muscle: Subject 4 chose the left biceps as muscle 1 and the left superior trapezius as muscle 2, whereas subject 5 chose the left superior and left middle trapezius, respectively, as 1 and 2. Muscle 1 contraction resulted in palmar grasping, whereas a contraction of muscle 2 resulted in lateral grasping (mode 5).

We randomly presented two distinct objects to subjects 4 and 5. They performed 11 hand grasping tests with the robot hand (Fig. 6). To grasp the objects, the subjects had to make either a palmar prehension via muscle 1 contraction, or a lateral prehension through muscle 2 contraction. Among the 11 trials, subject 4 had $100 \%$ success, while patient 5 managed to seize eight objects out of 11 . The three failures occurred with the palmar grasp because of co-contraction. Indeed, cocontraction was still present to some degree but this was the first muscle to reach the threshold that is considered to trigger the hand movement. Patient 5 tended to push the shoulder back (this activated the middle trapeze) just before raising it (this activated the superior trapezius).

\section{Comfort survey}

For protocol B (subjects 4-5), we present in Table $\mathrm{V}$ the responses of the subjects to the questionnaire on comfort and fatigue, related to the contraction of the different muscles. Each subject declared some muscles to be easier and more comfortable to contract (in terms of effort, fatigue, and concentration) than others.

\section{DISCUSSION}

The control of a neuroprosthesis by the user - that is, the patient - is a key issue, especially when the objective is to restore movement. Control should be intuitive and thus easily linked to task finality [4], [3], [12]. Furthermore, interfaces are based on the observation (i.e., sensing) of 
TABLE III

MUSCLE CONTRACTION ABILITIES. D: MAXIMUM CONTRACTION DURATION. ** FAVORITE MUSCLE, *WITH HELP OF ARM SUPPORT

\begin{tabular}{|c|c|c|c|c|c|c|c|c|}
\hline Subject ID & \multicolumn{2}{|l|}{ superior trapezius } & \multicolumn{2}{|c|}{$\begin{array}{l}\text { middle deltoid / } \\
\text { middle trapezius }\end{array}$} & \multicolumn{2}{c|}{ biceps } & \multicolumn{2}{c|}{ platysma } \\
\hline & Right (I) & Left (C) & Right (I) & Left (C) & Right (I) & Left (C) & Right (I) & Left (C) \\
\hline 1 & $10 \mathrm{~s} * *$ & NA & $>15 \mathrm{~s}$ & NA & 0 & NA & $>15 \mathrm{~s}$ & NA \\
\hline 2 & $>15 \mathrm{~s}^{* *}$ & $>15 \mathrm{~s}$ & $>15 \mathrm{~s}$ & $>15 \mathrm{~s}$ & $>15 \mathrm{~s}$ & $>15 \mathrm{~s}$ & $>15 \mathrm{~s}$ & $>15 \mathrm{~s}$ \\
\hline 3 & $>15 \mathrm{~s}$ & NA & $>15 \mathrm{~s}$ & NA & $>15 \mathrm{~s}$ & NA & $>15 \mathrm{~s}$ & $>15 \mathrm{~s}$ \\
\hline 4 & $>15 \mathrm{~s} *$ & $>15 \mathrm{~s} *$ & $>15 \mathrm{~s}$ & $>15 \mathrm{~s}^{*}$ & $7 \mathrm{~s}$ & $>15 \mathrm{~s}^{* *}$ & $>15 \mathrm{~s}$ & $>15 \mathrm{~s}$ \\
\hline 5 & $>15 \mathrm{~s}$ & $15 \mathrm{~s}$ & $>15 \mathrm{~s}$ & $>15 \mathrm{~s}^{* *}$ & $>15 \mathrm{~s}$ & $>15 \mathrm{~s}^{* *}$ & $14 \mathrm{~s}$ & $>15 \mathrm{~s}$ \\
\hline
\end{tabular}

TABLE IV

ABILITY TO GRADE THE CONTRACTION FOR THE 3 FIRST SUBJECTS, TIME FOR EACH CONTRACTION: 5 S (PROTOCOL A)

\begin{tabular}{|c|c|c|c|c|c|c|c|c|c|c|c|c|c|}
\hline & \multirow{2}{*}{$\begin{array}{c}\text { Level } \\
\text { of } \\
\text { contraction }\end{array}$} & \multicolumn{3}{|c|}{ upper Trapezius } & \multicolumn{3}{|c|}{ middle Deltoid } & \multicolumn{3}{|c|}{ Biceps } & \multicolumn{3}{|c|}{ Platysma } \\
\hline & & $\begin{array}{c}\text { Average } \\
(\mathrm{mV})\end{array}$ & $\begin{array}{l}\text { STD } \\
(\mathrm{mV})\end{array}$ & $\begin{array}{c}\text { Normalised } \\
\text { value }(\%)\end{array}$ & $\begin{array}{c}\text { Average } \\
\mathrm{mV})\end{array}$ & $\begin{array}{l}\text { STD } \\
(\mathrm{mV})\end{array}$ & $\begin{array}{c}\text { Normalised } \\
\text { value }(\%)\end{array}$ & $\begin{array}{c}\text { Average } \\
(\mathrm{mV})\end{array}$ & $\begin{array}{l}\text { STD } \\
(\mathrm{mV})\end{array}$ & $\begin{array}{l}\text { Normalised } \\
\text { value }(\%)\end{array}$ & $\begin{array}{c}\text { Average } \\
\mathrm{mV})\end{array}$ & $\begin{array}{l}\text { STD } \\
(\mathrm{mV})\end{array}$ & $\begin{array}{c}\text { Normalised } \\
\text { value }(\%)\end{array}$ \\
\hline \multirow{3}{*}{ Subject 1} & 1 & 75.33 & 18.87 & 0.32 & 72.93 & 9.51 & 0.6 & NA & NA & NA & 53.7 & 16.88 & 0.39 \\
\hline & 2 & 104 & 12.9 & 0.44 & 84.53 & 10.53 & 0.69 & NA & NA & NA & 59.83 & 14.3 & 0.44 \\
\hline & 3 & 237 & 59.9 & 1 & 122.13 & 12.87 & 1 & NA & NA & NA & 135.83 & 19.4 & 1 \\
\hline \multirow{3}{*}{ Subject 2} & 1 & 50.94 & 7.81 & 0.22 & 273.3 & 59.9 & 0.52 & 110.9 & 8.07 & 0.39 & 73.7 & 2 & 0.35 \\
\hline & 2 & 96.36 & 3.87 & 0.42 & 370 & 73.2 & 0.71 & 164.8 & 30.8 & 0.58 & 157.5 & 14.5 & 0.74 \\
\hline & 3 & 226.97 & 211.51 & 1 & 522 & 61.1 & 1 & 285.8 & 50.5 & 1 & 213 & 51.6 & 1 \\
\hline \multirow{3}{*}{ Subject 3} & 1 & 53.93 & 19.32 & 0.29 & 85.42 & 5 & 0.25 & 21.38 & 6.39 & 0.37 & 42.5 & 11.19 & 0.30 \\
\hline & 2 & 116.32 & 38.11 & 0.63 & 185 & 33.75 & 0.54 & 41.5 & 8.74 & 0.72 & 100 & 15.11 & 0.70 \\
\hline & 3 & 185 & 56.05 & 1 & 345 & 72.25 & 1 & 57.38 & 10.21 & 1 & 143.61 & 32.58 & 1 \\
\hline
\end{tabular}

TABLE V

EVALUATION OF INDIVIDUAL MUSCLE CONTRACTION FOR SUBJECTS 4 AND 5 (PROTOCOL B), * 1=VERY HIGH EFFORTS AND FATIGUE, $7=$ VERY LOW EFFORTS AND FATIGUE

\begin{tabular}{|c|c|c|c|c|c|c|c|c|c|c|c|c|c|c|c|c|}
\hline & \multicolumn{4}{|c|}{$\begin{array}{l}\text { Superior } \\
\text { trapezius }\end{array}$} & \multicolumn{4}{|c|}{$\begin{array}{l}\text { Middle deltoid / } \\
\text { Middle trapezius }\end{array}$} & \multicolumn{4}{|c|}{ Biceps } & \multicolumn{4}{|c|}{ Platysma } \\
\hline & \multicolumn{2}{|c|}{ Right } & \multicolumn{2}{|c|}{ Left } & \multicolumn{2}{|c|}{ Right } & \multicolumn{2}{|c|}{ Left } & \multicolumn{2}{|c|}{ Right } & \multicolumn{2}{|c|}{ Left } & \multicolumn{2}{|c|}{ Right } & \multicolumn{2}{|c|}{ Left } \\
\hline & Comfort & Fatigue & Comfort & Fatigue & Comfort & Fatigue & Comfort & Fatigue & Comfort & Fatigue & Comfort & Fatigue & Comfort & Fatigue & Comfort & Fatigue \\
\hline Subject 4 & 3.8 & 2 & 3 & 2 & 4.5 & 2.5 & 3.3 & 5.3 & 4.3 & 3.7 & 5 & 5.7 & 2.25 & 2.5 & 3 & 2 \\
\hline Subject 5 & 7 & 4.3 & 2.5 & 1 & 6.8 & 6.3 & 4 & 3 & 2.5 & 5 & 3.5 & 6.3 & 2 & 1 & 3.5 & 3 \\
\hline
\end{tabular}

voluntary actions (even mentally imagined, as with BCI interfaces [9]). EMG is widely used to achieve this goal for amputees, but for patients with tetraplegia, the use of supra-lesional muscles to control infra-lesional muscles was a neat option. The second generation of the Freehand system was successfully developed and is the only implanted EMGcontrolled neuroprosthesis to date. As far as we know, robot hands for tetraplegics have not yet been controlled using EMG.

The feasibility of using supra-lesional muscle EMG was not straightforward. Indeed, the available muscles are few and most of them cannot be considered valid as they are underused and their motor schema is in some cases deeply impaired, with no functional output. This leads to highly fatigable and weak muscles, but also to the loss of synergy between the paralyzed muscles that are normally involved in upper limb movements. In some cases, even if the muscle is contractable, the produced contraction is not functional (does not induce any joint motion).

Here, the goal was to understand whether the immediately supra-lesional muscles of tetraplegic patients could be used to control a robot hand. The targeted population - that is, tetraplegics with potentially weak supra-lesional muscles should have a very simple interface for two reasons: (i) simple contraction schemes to control the hand limit cognitive fatigue, and (ii) short contractions limit physiological fatigue. These two constraints mean that the hand should be controlled with predefined postures and not in a proportional way. Thus, the output of our control framework was a limited set of hand states, while its input, except for one mode (mode 3), was a limited set of EMG levels. In this context, the FSM scheme should be preferred. In our study, we found in all five subjects a combination of muscles such that each was able to easily perform the tasks (protocol A) that is, to maintain a 

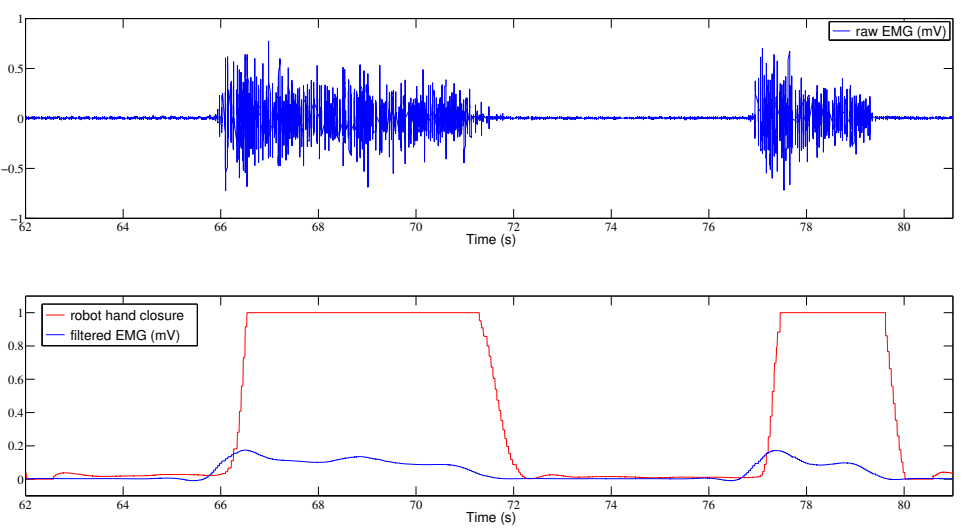

(a) Mode 1
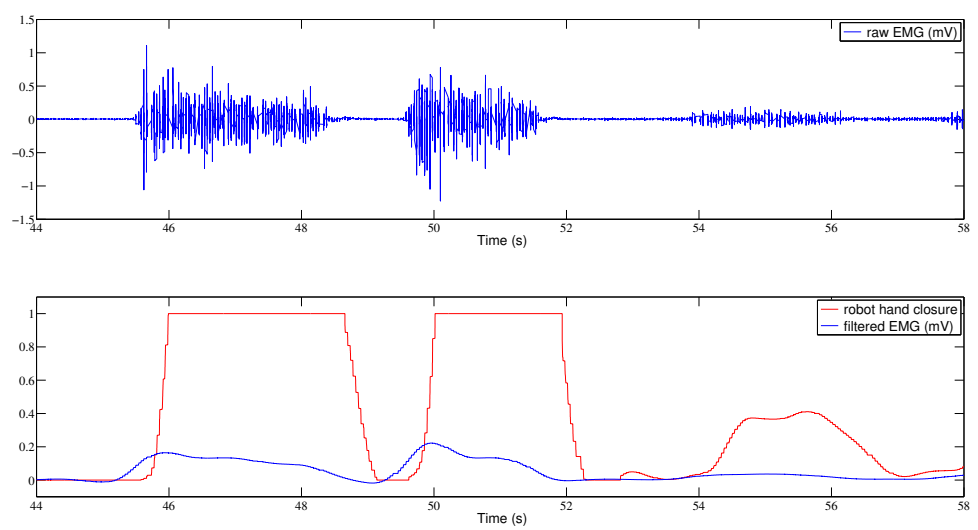

(b) Mode 3

Fig. 6. Example of robot hand trajectories generated from EMG recording in subject 5 for modes 1 and 3. Top: raw EMG, Bottom: filtered EMG (blue) and hand trajectory (red).0: hand is open, 1 : hand is closed.

continuous contraction or a grade contraction, so that it could be quantified by an EMG signal. We were able to calibrate quite low thresholds, so that patients did not have to contract much and experience fatigue. Moreover, these experiments were conducted during the scheduled clinical assessment, so no training was offered, even during the session. The patients were merely asked to contract muscles and to try to hold objects with the robot hand. All were able to control it immediately. The calibration procedure is linked only to EMG signal scaling so that, as a whole, the system is very easy to use in a clinical context, compared with approaches like BCI, for instance. Interestingly, the lesion age had no influence on performance.

Two subjects participated in the second session (protocol B), in which the EMG signals were used to control a robot hand. This was achieved without any prior learning or training. We show that both the used muscle, and the way the contraction controls the hand (control mode), have a drastic effect on performance. This robot hand approach may thus be a very good paradigm for rehabilitation or training, for future FES-based control of the patients' own hand.

These two subjects did not have the same preferred mode of control, but clearly preferred one over the others. Mode 1 (continuous contraction to maintain robot hand closure) seems to be more intuitive, as the contraction is directly linked to the posture of the hand, but mode 2 (an impulsive contraction provokes robot hand closure/opening) induces less fatigue as it needs only short muscle contractions to toggle from open to closed hand. Depending on their remaining motor functions, patients feel more or less comfortable with a given mode. Also, the choice of the preferred control mode would probably be different after a training period. In our opinion, patients should select their preferred mode themselves. However, a larger study would give indications on how to classify patients preferred modes, based on the assessment of their muscle state. In any case, control cannot be defined through a single mode and should be adapted to each patient and probably to each task and fatigue state.

For practical reasons, we decided that the two EMGs would be located on the same side without any knowledge beforehand as to which side to equip. The subject selected one preferred muscle and based on this choice, the second muscle was selected on the same side. A major issue with this decision is that the two muscles sometimes co-contract and in mode 5 (muscle 1 contraction causes palmar pinch and muscle 2 contraction causes key-grip) the robot hand grasping task selected by the system was not always the one the user intended to execute.

In the future, patients will control their own hand by means of electrical stimulation instead of a distant robot hand, and the choice of which body side to equip with EMG will need to be made with respect to the task that the stimulated hand must achieve. For example, if muscle contraction is associated with arm motion, this might well disturb the grasping to be achieved. Furthermore, an analysis is needed to determine the effect of the dominant side on performance.

For our patients, grasping would not be disturbed since shoulder movements do not induce forearm movements. The questionnaire at the end of each test allowed us to evaluate the ease of using EMG as a control method. Preferential muscles were chosen so as not to disturb the functionalities available to the subjects. Yet, one can also imagine a system that deactivates electrostimulation when the patient wishes to use his/her remaining functionality for other purposes. In this case, the subject would be able to contract his/her muscles without causing hand movements. Furthermore, one can imagine using forearm/arm muscle synergies or relevant motor schemas to facilitate the learning (e.g. hand closing when the elbow bends, hand opening during elbow extension, and so on).

The interesting property of the proposed interface is that even a weak muscle can produce a proper EMG signal. As an example, subject 4 was able to control the robot hand with a weak muscle to produce functional movement. In other words, a non-functional muscle in the context of natural movements can be turned into a functional muscle in the context of assistive technology and one can even expect that motor performances will improve with training. 


\section{CONCLUSION}

We have demonstrated the feasibility of extracting contraction recordings from supra-lesional muscles in individuals with tetraplegia that are sufficiently rich in information to pilot a robot hand. The choice of muscles and modes of control are patient-dependent. Any available contractable muscle and not just functional muscles - can be candidates and should be evaluated. The control principle could also be used for FES applied to the patient arm, or to control an external device such as a robot arm or electric wheelchair, or as a template of rehabilitation movements. The robot hand might help to select (via their residual control capacity), and possibly train, patients as potential candidates for an implanted neuroprosthetic device. A greater number of patients using the robot hand would provide a better picture of the range of performance. Therefore, the next step will be to extend the study to a wider group of patients, to provide a better picture of the range of performance. We also plan to use the robot hand as a part of a training protocol for future FES devices.

\section{ACKNOWLEDGMENTS}

The authors wish to thank the subjects who invested time into this research, as well as MXM-Axonic/ANRT for support with the $\mathrm{PhD}$ grant, CIFRE \# 2013/0867. The work was also supported in part by the ANR (French National Research Agency) SISCob project ANR-14-CE27-0016. Last, the authors also warmly thank Violaine Leynaert, occupational therapist at Propara Center, for her precious help.

\section{REFERENCES}

[1] M. W. Keith, P. H. Peckham, G. B. Thrope, K. C. Stroh, B. Smith, J. R. Buckett, K. L. Kilgore, and J. W. Jatich, "Implantable functional neuromuscular stimulation in the tetraplegic hand," The Journal of hand surgery, vol. 14, no. 3, pp. 524-530, 1989.

[2] C. Billian and P. H. Gorman, "Upper extremity applications of functional neuromuscular stimulation," Assistive Technology, vol. 4, no. 1, pp. 3139, 1992.

[3] M. W. Keith, "Neuroprostheses for the upper extremity," Microsurgery, vol. 21, no. 6, pp. 256-263, 2001.

[4] N. Hoshimiya, a. Naito, M. Yajima, and Y. Handa, "A multichannel FES system for the restoration of motor functions in high spinal cord injury patients: a respiration-controlled system for multijoint upper extremity." IEEE transactions on bio-medical engineering, vol. 36, no. 7, pp. 754-60, Jul. 1989. [Online]. Available: http: //www.ncbi.nlm.nih.gov/pubmed/2787283

[5] D. Guiraud, T. Stieglitz, K. Koch, J. Divoux, and P. Rabischong, "An implantable neuroprosthesis for standing and walking in paraplegia: 5year patient follow-up." J Neural Eng., vol. 3, no. 4, pp. 268-75, 2006.

[6] J. Jovic, C. Azevedo Coste, P. Fraisse, S. Henkous, and C. Fattal, "Coordinating Upper and Lower Body During FES-Assisted Transfers in Persons With Spinal Cord Injury in Order to Reduce Arm Support," Neuromodulation: Technology at the Neural Interface, vol. 18, p. 7, May 2015. [Online]. Available: http: //hal-lirmm.ccsd.cnrs.fr/lirmm-01146757

[7] C. W. Moss, K. L. Kilgore, and P. H. Peckham, "A novel command signal for motor neuroprosthetic control," Neurorehabilitation and neural repair, vol. 25, no. 9, pp. 847-854, 2011.

[8] Q. Zhang, M. Hayashibe, and C. Azevedo Coste, "Evoked electromyography-based closed-loop torque control in functional electrical stimulation,” IEEE Trans Biomed Eng., vol. 60, no. 8, pp. 2299-307, 2013.

[9] C. King, P. Wang, C. McCrimmon, C. Chou, A. Do, and Z. Nenadic, "The feasibility of a brain-computer interface functional electrical stimulation system for the restoration of overground walking after paraplegia." Journal of NeuroEngineering and Rehabilitation, vol. 3, no. 4, pp. 26875, 2015.
[10] J. S. Knutson, H. A. Hoyen, K. L. Kilgore, and P. H. Peckham, "Simulated neuroprosthesis state activation and hand-position control using myoelectric signals from wrist muscles." J Rehabil Res Dev, vol. 41, no. 3B, pp. 461-72, 2004.

[11] R. L. Hart, K. L. Kilgore, and P. H. Peckham, "A comparison between control methods for implanted fes hand-grasp systems," Rehabilitation Engineering, IEEE Transactions on, vol. 6, no. 2, pp. 208-218, 1998.

[12] N. Bhadra, P. H. Peckham, M. W. Keith, K. L. Kilgore, F. Montague, M. Gazdik, and T. Stage, "Implementation of an implantable joint-angle transducer," Journal of rehabilitation research and development, vol. 39, no. 3, pp. 411-422, 2002.

[13] T. Keller, A. Curt, M. R. Popovic, A. Signer, and V. Dietz, "Grasping in high lesioned tetraplegic subjects using the EMG controlled neuroprosthesis," NeuroRehabilitation-An Interdisciplinary Journal, vol. 10, no. 3, pp. 251-256, 1998.

[14] R. Thorsen, D. Dalla Costa, S. Chiaramonte, L. Binda, E. Beghi, T. Redaelli, E. Occhi, and M. Ferrarin, "A noninvasive neuroprosthesis augments hand grasp force in individuals with cervical spinal cord injury: The functional and therapeutic effects," The Scientific World Journal, vol. 2013, 2013

[15] W. D. Memberg, K. H. Polasek, R. L. Hart, A. M. Bryden, K. L. Kilgore, G. A. Nemunaitis, H. A. Hoyen, M. W. Keith, and R. F. Kirsch, "Implanted neuroprosthesis for restoring arm and hand function in people with high level tetraplegia," Archives of physical medicine and rehabilitation, vol. 95, no. 6, pp. 1201-1211, 2014.

[16] M. DiCicco, L. Lucas, and Y. Matsuoka, "Comparison of control strategies for an EMG controlled orthotic exoskeleton for the hand," in IEEE Int. Conf. on Robotics and Automation, ICRA, vol. 2, 2004, pp. 1622-1627.

[17] W. Tigra, C. Azevedo Coste, C. Fattal, and D. Guiraud, "Ergonomics of the control by a quadriplegic of hand functions," in Neural Engineering (NER), 7th Int. IEEE/EMBS Conf. on, 2015, pp. 759-762.

[18] M. Cutkosky, "On grasp choice, grasp models, and the design of hands for manufacturing tasks," Robotics and Automation, IEEE Trans. on, vol. 5, no. 3, pp. 269-279, Jun 1989.

[19] A. Bicchi, "Hands for dexterous manipulation and robust grasping: a difficult road toward simplicity," Robotics and Automation, IEEE Trans. on, vol. 16, no. 6, pp. 652-662, Dec 2000.

[20] J. Weisz, C. Elvezio, and P. K. Allen, "A user interface for assistive grasping," in IEEE/RSJ Int. Conf. on Intelligent Robots and Systems, IROS, 2013

[21] K. Farry, I. Walker, and R. Baraniuk, "Myoelectric teleoperation of a complex robotic hand," Robotics and Automation, IEEE Trans. on, vol. 12 , no. 5 , pp. $775-788$, Oct 1996 .

[22] L. Zollo, S. Roccella, E. Guglielmelli, M. Carrozza, and P. Dario, "Biomechatronic design and control of an anthropomorphic artificial hand for prosthetic and robotic applications," Mechatronics, IEEE/ASME Trans. on, vol. 12, no. 4, pp. 418-429, Aug 2007.

[23] C. Cipriani, F. Zaccone, S. Micera, and M. Carrozza, "On the shared control of an EMG-controlled prosthetic hand: Analysis of user," Robotics, IEEE Trans. on, vol. 24, no. 1, pp. 170-184, Feb 2008.

[24] B. A. Kent, N. Karnati, and E. D. Engeberg, "Electromyogram synergy control of a dexterous artificial hand to unscrew and screw objects," Journal of NeuroEngineering and Rehabilitation, vol. 11, no. 41, 2014.

[25] L. R. Hochberg, D. Bacher, B. Jarosiewicz, N. Y. Masse, J. D. Simeral, J. Vogel, S. Haddadin, J. Liu, S. S. Cash, P. Van der Smagt, and J. P. Donoghue, "Reach and grasp by people with tetraplegia using a neurally controlled robotic arm," Nature, vol. 485, no. 7398, pp. 372-375, 2012.

[26] G. Pfurtscheller, G. R. Müller, J. Pfurtscheller, H. J. Gernerd, and R. Rupp, "Thought control of functional electrical stimulation to restore hand grasp in a patient with tetraplegia," Neuroscience Letters, vol. 351, no. 1, pp. 33-36, 2003.

[27] S. A. Dalley, H. A. Varol, and M. Goldfarb, "A method for the control of multigrasp myoelectric prosthetic hands," Neural Systems and Rehabilitation Engineering, IEEE Transactions on, vol. 20, no. 1, pp. 58-67, 2012.

[28] S. C. Kirshblum, S. P. Burns, F. Biering-Sorensen, W. Donovan, D. E. Graves, A. Jha, M. Johansen, L. Jones, A. Krassioukov, M. Mulcahey et al., "International standards for neurological classification of spinal cord injury (revised 2011)," The journal of spinal cord medicine, vol. 34 no. 6, pp. 535-546, 2011. 